Article

\title{
Overview of a Theory for Planning Similar Experiments with Different Fluids at Supercritical Pressure
}

\author{
Andrea Pucciarelli (D), Sara Kassem and Walter Ambrosini *(D) \\ Dipartimento di Ingegneria Civile e Industriale, Università di Pisa, Largo Lucio Lazzarino 2, 56122 Pisa, Italy; \\ andrea.pucciarelli@dici.unipi.it (A.P.); sara.kassem@phd.unipi.it (S.K.) \\ * Correspondence: walter.ambrosini@unipi.it
}

Citation: Pucciarelli, A.; Kassem, S.; Ambrosini, W. Overview of a Theory for Planning Similar Experiments with Different Fluids at Supercritical Pressure. Energies 2021, 14, 3695. https://doi.org/10.3390/en14123695

Academic Editors: Rosa Lo Frano and Guglielmo Lomonaco

Received: 20 May 2021

Accepted: 17 June 2021

Published: 21 June 2021

Publisher's Note: MDPI stays neutral with regard to jurisdictional claims in published maps and institutional affiliations.

Copyright: (C) 2021 by the authors. Licensee MDPI, Basel, Switzerland. This article is an open access article distributed under the terms and conditions of the Creative Commons Attribution (CC BY) license (https:// creativecommons.org/licenses/by/ $4.0 /)$.

\begin{abstract}
The recent advancements achieved in the development of a fluid-to-fluid similarity theory for heat transfer with fluids at supercritical pressures are summarised. The prime mover for the development of the theory was the interest in the development of Supercritical Water nuclear Reactors (SCWRs) in the frame of research being developed worldwide; however, the theory is general and can be applied to any system involving fluids at a supercritical pressure. The steps involved in the development of the rationale at the basis of the theory are discussed and presented in a synthetic form, highlighting the relevance of the results achieved so far and separately published elsewhere, with the aim to provide a complete overview of the potential involved in the application of the theory. The adopted rationale, completely different from the ones in the previous literature on the subject, was based on a specific definition of similarity, aiming to achieve, as much as possible, similar distributions of enthalpies and fluid densities in a duct containing fluids at a supercritical pressure. This provides sufficient assurance that the complex phenomena governing heat transfer in the addressed conditions, which heavily depend on the changes in fluid density and in other thermophysical properties along and across the flow duct, are represented in sufficient similarity. The developed rationale can be used for planning possible counterpart experiments, with the aid of supporting computational fluid-dynamic (CFD) calculations, and it also clarifies the role of relevant dimensionless numbers in setting up semi-empirical correlations for heat transfer in these difficult conditions, experiencing normal, enhanced and deteriorated regimes. This paper is intended as a contribution to a common reflection on the results achieved so far in view of the assessment of a sufficient body of knowledge and understanding to base successful predictive capabilities for heat transfer with fluids at supercritical pressures.
\end{abstract}

Keywords: supercritical water; nuclear reactors; heat transfer; SCWR; similarity

\section{Introduction}

Fluids at supercritical pressures exhibit a complex variety of convective heat transfer behaviours, ranging from "normal heat transfer", similar to the one observed in fluids with little property changes, to "enhanced (or improved) heat transfer", mainly caused by the larger specific heat occurring at a pressure-dependent temperature threshold defined as "pseudocritical", to "deteriorated heat transfer", occurring owing to a combination of factors mainly depending on the fluid property changes. These phenomena were observed in early experiments and were explained in classical and more recent works (see, e.g., [1,2]) on the basis of considerations related to the consequent fluid velocity and property distribution changes in the bulk fluid and close to the heating wall.

In this frame, the proposed design of nuclear reactors cooled and moderated by supercritical pressure water (SCWRs) had a propulsive effect on the knowledge and understanding, stimulating efforts to collect available information and propose it in a convenient form for adoption in an environment in which safety is an overriding priority (see references [3-9]). These efforts also take into account the experiences from applications in the 
field of fossil-fuelled plants, though the safety requirements typical of the nuclear field stimulated to a better accuracy and improved understanding of the underlying phenomena.

Similarity theories for comparing the observed experimental trends in a fluid-to-fluid perspective were proposed in several steps; some of them were summarised in recent papers $[10,11]$ also mentioning the first proposals made by these authors in previous publications. In general terms, the existing similarity theories were not considered completely satisfactory [11], though they were often taken into account to provide first guesses in designing experiments with simulant fluids, hoping for some degree of similarity with respect to the conditions to be really experimented on with water in nuclear reactor operations.

The rationale adopted by the authors and summarised in this paper is based on ideas firstly applied in the analysis of the flow stability of heated channels with fluids at supercritical pressure [12], adapted to achieve first, embryonal choices about the main parameters playing a role in heat transfer [13]. At that time, the idea was introduced to support the theoretical considerations at the basis of the first proposed rationale by the use of CFD calculations as an advanced methodology to get information about the potential and challenges involved in the theory. In fact, using experimental data to support the theory would require finding in previous works occasionally matching boundary conditions for different fluids or to set up purposely developed experimental campaigns. The use of CFD models was, at that time, highly unreliable, since the deficiencies in two-equation turbulence models were well-known, with $k-\varepsilon$ models showing a tendency to overestimate heat transfer deterioration, while, in many instances, $\mathrm{k}-\omega$ models showed some degree of insensitivity to the onset of heat transfer deterioration $[14,15]$. This situation could not be considered satisfactory, and subsequent works concentrated on the assessment and the development of more reliable turbulence models; this effort was rewarded by better predictions at least in some operating ranges [16-20] owing to the use of an algebraic heat flux model (AHFM) and to its implementation in a low-Re turbulence model adopted in a commercial code [21].

Owing to these new capabilities and to the higher reliability of obtained predictions, a better confidence was assigned to the CFD models, so that their use in supporting the basis of a similarity theory was fully justified. This resulted in a thorough revision of the similarity principles and in their application to some exemplary cases having as reference experimental and DNS data obtained with water and carbon dioxide [22,23]. In these applications, the adopted methodology consisted of a first step in which experimental or DNS data were predicted by the RANS turbulence model, assuring a sufficient coherence in the prediction of phenomena, then evaluating the postulated behaviour of the other fluids (namely, water, carbon dioxide, ammonia and R23). The predicted phenomena were assessed both in dimensional and dimensionless forms, in order to assure that both qualitative and quantitative features of the bulk and wall temperature trends were sufficiently well-represented.

In brief, this rationale requests, firstly, a check of the RANS model in front of reliable experimental data and, then, the extrapolation to other fluids with "similar" boundary conditions. This methodology was used in the latest publications [24-26], which addressed both $\mathrm{CO}_{2}$ and water experimental data [27,28], as well as DNS data proposed by an independent group [29], aiming to check the early principles set forth in reference [13]. On the basis of the obtained success in reproducing similar trends with four different fluids, further developments were planned and partly already proposed to the scientific community in recently published and shortly forthcoming papers [30-33].

The present paper has the purpose of summarising the achieved results in a comprehensive way, starting from the first motivations for the work performed up to now, and to discuss the achievements obtained in its steps. Since the theory may be considered for planning confirmatory experiments, independent checks are expected to be made by other research groups in order to possibly confirm and refine the similarity principles at the basis of the present rationale. 


\section{Heat Transfer to Supercritical Pressure Fluids and Its Challenges}

The trends of the fluid properties at supercritical pressures for the two relevant fluids shown in Figures 1 and 2, water at $25 \mathrm{MPa}$ as the coolant proposed for most SCWR concepts and used in the experimental data by Watts [27] and $\mathrm{CO}_{2}$ at $8.35 \mathrm{MPa}$, adopted in some of the experiments taken into account in a recent work [28], justify the complexities exhibited by the observed heat transfer phenomena.

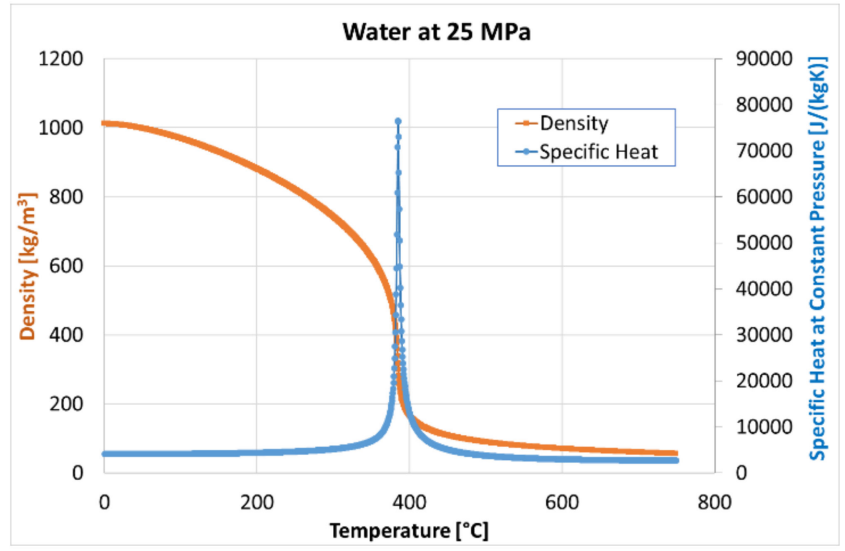

(a)

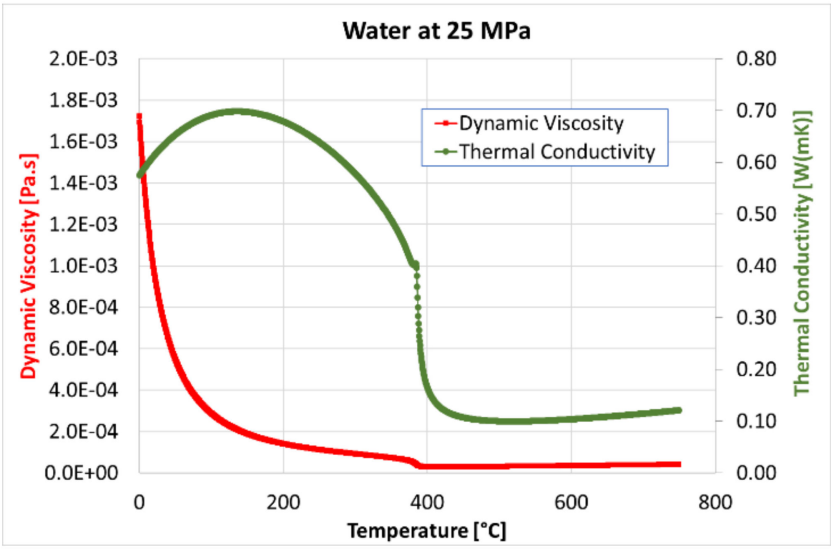

(b)

Figure 1. Thermodynamic (a) and transport properties (b) for water at $25 \mathrm{MPa}$.

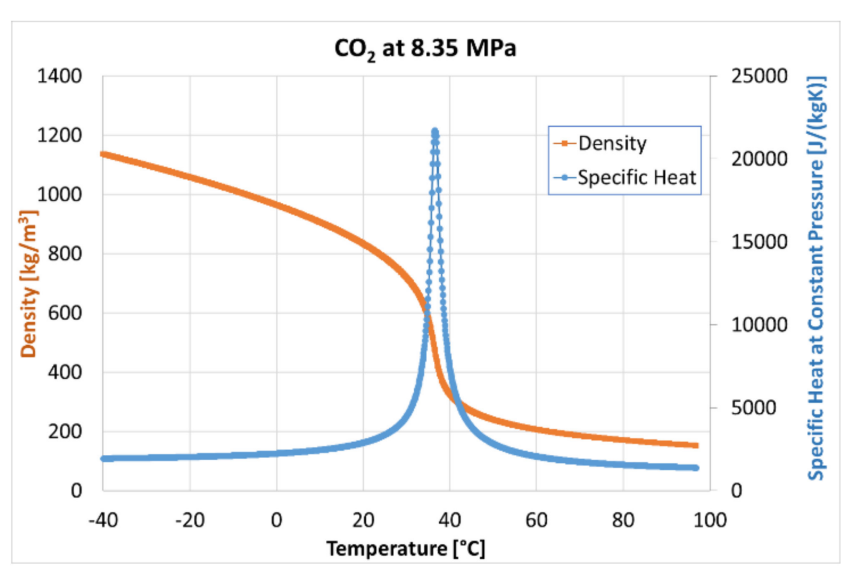

(a)

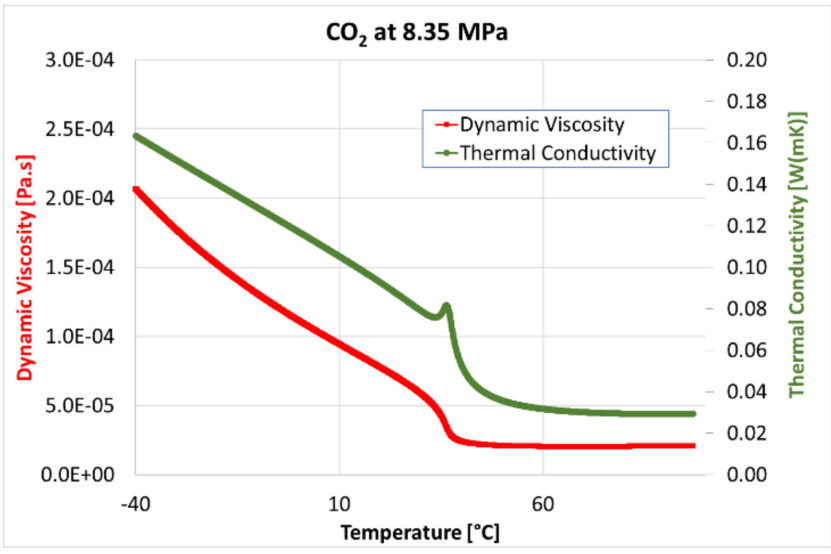

(b)

Figure 2. Thermodynamic (a) and transport properties $(\mathbf{b})$ of carbon dioxide at $8.35 \mathrm{MPa}$.

Indeed, for the operating conditions spanning from the liquid-like to the gas-like region, i.e., through the pseudocritical temperature, at which a specific heat experiences a peak, the changes in the fluid properties appear quite dramatic. Acceleration and buoyancy phenomena do occur inside a vertical flow duct, owing to the huge density changes along and across the channel, caused by the variation of the temperature.

Figure 3 describes one such case previously analysed by the authors in reference [20] making use of CFD, based on experimental data by Kline [28] obtained with carbon dioxide. In agreement with the experiment (comparisons are shown in reference [20]), the code predicts the occurrence of deteriorated heat transfer (DHT) soon after the entrance of the heated length in the test section (some $30 \mathrm{~cm}$ from the pipe inlet). In the downstream part of the pipe, the conditions are encountered for heat transfer restoration, with a subsequent increase of the turbulence. 


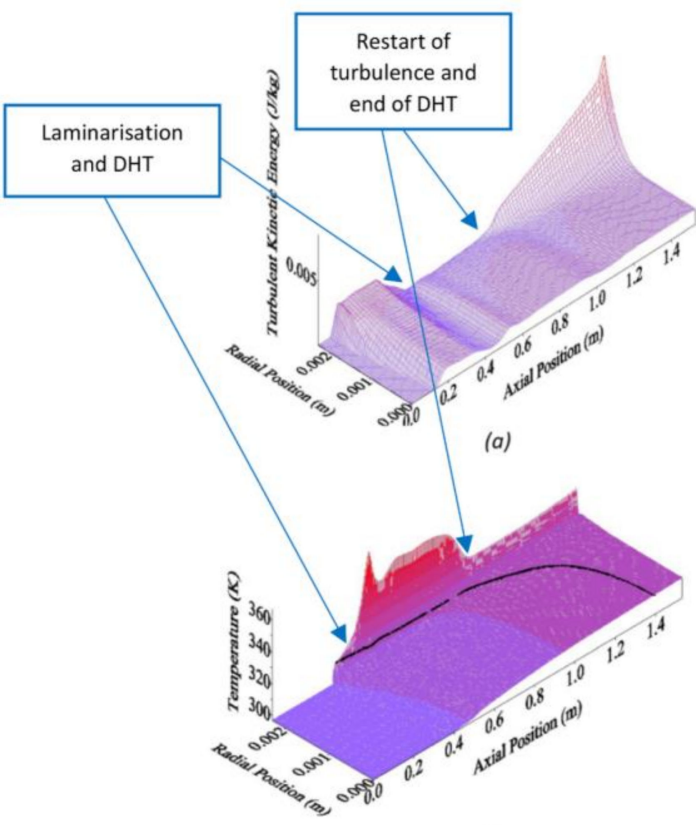

(c)

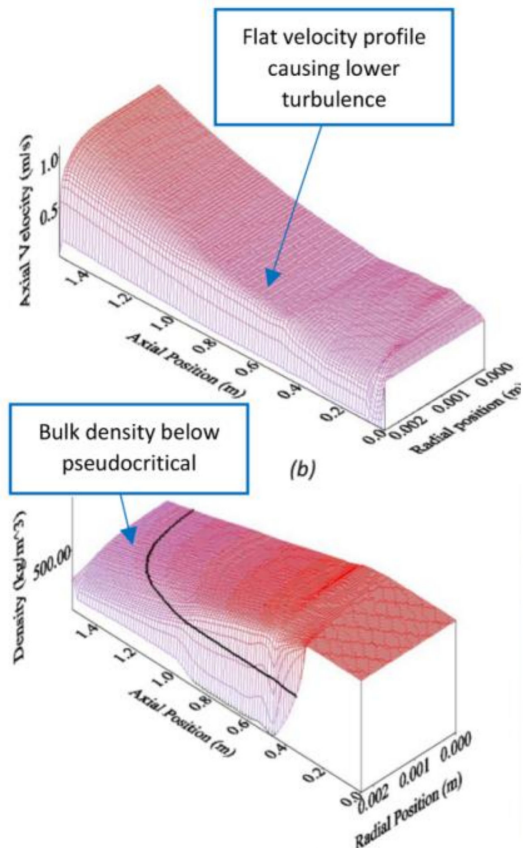

(d)

Figure 3. Sequence of changes in the turbulent kinetic energy (a), axial velocity (b), fluid temperature (c) and density (d) predicted by CFD for some of Kline's [28] boundary conditions $\left(\mathrm{CO}_{2}\right.$ at $\left.8.35 \mathrm{MPa}\right)$ (adapted from reference [20] with Editor's permission).

A few interesting features shown in Figure 3 are worth commenting on. Downstream the inlet section, at which a flat velocity distribution is assumed, the radial velocity profile (Figure $3 b$ ) adjusts on a typically turbulent shape until the heated section is entered, causing the fluid density to decrease close to the wall (Figure 3d). This decrease generates a flow redistribution that results in flattening the velocity profile close to the wall, reducing the shear stress and the production of turbulent kinetic energy (Figure 3a). The oscillations observed in the wall temperature (Figure 3c), clearly explained in reference [34] in their general features, have their root in the corresponding waves observed on the surface representing the turbulent kinetic energy (Figure 3a), caused by tiny oscillations in the slope of the radial velocity distribution, which affect the turbulence production by shearing. Though they are difficult to detect in Figure 3b, they can be observed in detailed views and have counterparts in the corresponding waves in the turbulent kinetic energy (Figure 3a).

The black curves appearing on the surfaces in Figure 3c,d represent the loci of the pseudocritical conditions in both the fluid temperature and density. They show the reason for the occurrence of the final restoration of heat transfer, which happens when the density difference at the wall and in bulk becomes low enough, owing to the transition to a gas-like phase beyond the pseudocritical threshold, so that mixed convection effects cannot further decrease the turbulence production. As a consequence, the velocity profile is shown to recover a classical turbulent shape (see the last part of Figure 3b), and turbulent kinetic energy restarts its growth close to the wall (Figure 3a).

As can be noted, in the considered case the trend of density close to the wall and in the bulk fluid fully governs the heat transfer behaviour, determining the heat transfer deterioration, the damped temperature oscillations at the wall after deterioration and the final restoration of heat transfer. Other effects occurring simultaneously with the transition through the pseudocritical temperature introduce additional phenomena; in particular, the huge changes in the fluid thermal conductivity and viscosity definitely add additional features in the above description, which is in full agreement with the previous explanations about the mechanism causing heat transfer deterioration [1]. 
Since density is so relevant in determining the observed phenomena, the first considerations in view of the establishment of a fluid-to-fluid similarity theory directly addressed its behaviour [13], though with limited success. Actually, better fortune since the very beginning provided the description of flow instability phenomena by a single trend of dimensionless density as a function of dimensionless enthalpy [12,35-37]. This is because in the 1D flow dynamic equations with imposed heat flux at the wall the one for density represents the only requested state equation. By virtue of finding a nearly unique trend between the dimensionless density and dimensionless enthalpy, defined as

$$
\rho^{*}=\rho / \rho_{p c} \text { and } h^{*}=\left(h-h_{p c}\right) \beta_{p c} / C_{p, p c}
$$

for a broad range of supercritical pressures and, at a lower extent, for four different fluids (water, carbon dioxide, ammonia and R23), it was possible to set up relatively universal stability maps as a function of the dimensionless parameters. This unique trend found to hold approximately for the four addressed fluids is reported in Figure 4. As it can be noted, this trend shows some deviations in the liquid-like region depending on the nature of the fluid; these deviations were recently observed to be larger for some other fluids of interest for experimental facilities (namely, R134a), though the similarity theory developed herein was recently shown to also apply with sufficient accuracy in that case [33]. It can be recognised then that this link between density and enthalpy at supercritical pressures is very appealing for establishing fluid-to-fluid similarity theories: indeed, this has been the basis for the rationale here in this discussion.

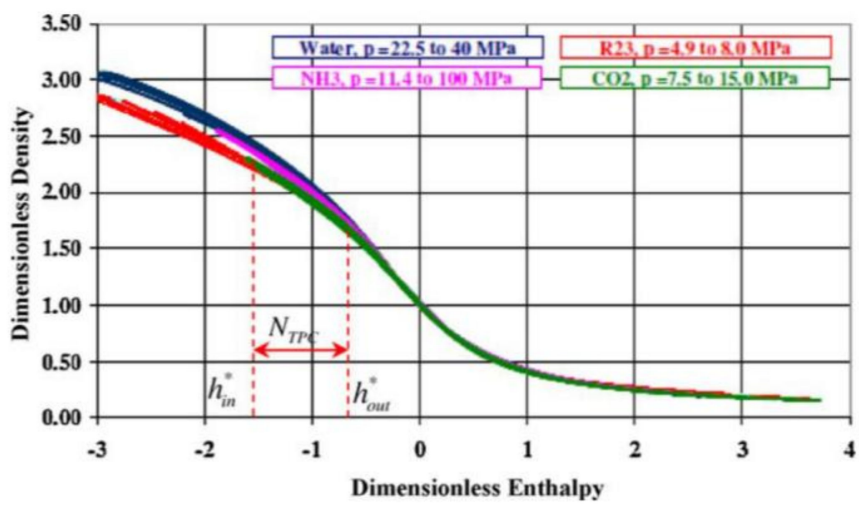

Figure 4. Trend of the dimensionless density vs. dimensionless enthalpy for four different fluids (adapted from reference [37] with the editor's permission).

\section{Adopted Fluid-to-Fluid Similarity Rationale}

Owing to the overwhelming importance of the density distribution within the flow duct in view of the establishment of different heat transfer regimes, it is considered of primary importance to preserve in similarity the density distribution along and across the flow duct. In particular, the capability of the fluid to expand under heating is considered as an aspect to be strictly preserved in view of a sufficiently accurate similarity. Considering the above-mentioned results about the dependence of dimensionless density with respect to dimensionless enthalpy, a direct consequence of this choice is the requirement of preserving the distribution of dimensionless enthalpy, again along and across the channel. The latter was therefore the leading consideration that guided the establishment of the rationale; some straightforward consequences of this choice are suggested in the following sections.

In order to preserve similar capabilities of fluid expansion when heated, the same dimensionless enthalpy (and, thus, density) must be kept at the inlet; in fact, the capability of a fluid to expand is critically dependent on its subcooling with respect to the pseudocritical 
threshold. This requires imposing that the same values of the sub-pseudocritical number be assumed for the different fluids, i.e.,

$$
N_{S P C}=-h_{i n}^{*}
$$

Since the bulk fluid expansion is dependent on the heating power-to-flow ratio, coherently with the definition of the dimensionless enthalpy, also the trans-pseudocritical number must be preserved for the two similar conditions:

$$
N_{T P C}=\frac{\dot{Q}}{W} \frac{\beta_{p c}}{C_{p, p c}}=h_{o u t}^{*}-h_{i n}^{*}
$$

(Note that, in the adopted notation variables without the subscript " $\mathrm{w}$ ", indicating wall, are assumed to be defined as bulk ones.). This assures that the bulk dimensionless enthalpy and density will have the same trends along the channel; though, as will be shown later, the length over the diameter ratio may change. Since the buoyancy forces were found so important, a basic requirement is that the Froude number and the Richardson number are nearly the same all along the duct. In this regard, two fortunate consequences of the previous assumptions were found:

- $\quad$ by imposing a same value of the inlet Froude number, $F r_{i n}=w_{i n}^{2} /(g D)$, the equality of the distributions of the bulk fluid dimensionless densities for different fluids assures its preservation all along the channel; this can be demonstrated considering that $F r=w^{2} /(g D)=\left[w_{i n}^{2} /(g D)\right]\left[w^{2} / w_{i n}^{2}\right]=F r_{i n}\left[w^{2} / w_{i n}^{2}\right]$; since, in steady-state conditions for the constant cross-section area, it is $G=\rho w=$ const., it follows $F r=F r_{i n}\left[\rho_{i n}^{2} / \rho^{2}\right]=F r_{i n}\left[\rho_{i n}^{* 2} / \rho^{* 2}\right]$, predicting an increase of the Froude number with the bulk fluid expansion along the duct at a same level, whatever the chosen fluid;

- moreover, assuming also that the wall dimensionless enthalpy is the same all along the duct, as it is necessary from our definition of similarity, the Richardson number is also preserved; in fact, from its definition, $R i=G r_{\rho} / R e^{2}=\left[\left(\rho-\rho_{w}\right) g D\right] / \rho w^{2}$, it can be found that $R i=\left(\rho-\rho_{w}\right) /(\rho F r)=\left(\rho^{*}-\rho_{w}^{*}\right) /\left(\rho^{*} F r\right)$; therefore, the preservation of the Froude number along the channel and the equality of dimensionless densities in bulk and at the wall also assure the preservation of the Richardson number.

The latter aspect is very useful in the present context, since it allows preserving the same ratio of inertia over the buoyancy forces in mixed convection, which have so much relevance for determining the occurrence of the deteriorated heat transfer described in previous sections.

As an additional requirement, it was decided to impose the equality of the inlet Reynolds numbers for the different fluids, as suggested in reference [29]. Actually, this condition was not specified in the first form of this rationale published in reference [23], since at the time it was already recognised that there is no means of assuring that the Reynolds number equality will be preserved all along the channel, even if it is preserved at the inlet. In fact, the downstream evolution of the kinematic viscosity of the fluids can be expected to be different enough so that any equality is generally impossible to be achieved. However, imposing in some place along the channel an exact equality of the Reynolds number may help to keep the levels of turbulence sufficiently comparable; whether the inlet section is the best place to impose this equality is nevertheless a subject open to further discussion.

Imposing the equality of both the inlet Reynolds and Froude numbers has the consequence that the diameter of the pipe must be different for different fluids, according to the following formulations:

$$
\frac{w_{\text {in,fluid-2 }}}{w_{\text {in,fluid }-1}}=\left[\frac{v_{\text {in,fluid-2 }}}{v_{\text {in,fluid-1 }}}\right]^{1 / 3} \frac{D_{\text {fluid-2 }}}{D_{\text {fluid-1 }}}=\left[\frac{v_{\text {in }, \text { fluid }-2}}{v_{\text {in }, \text { fluid }-1}}\right]^{2 / 3}
$$


An interesting relationship was held in a dimensionless form for the Newton law of convection, which can be expressed as $N_{T P C}=\overline{S t}\left(h_{w}^{*}-h^{*}\right) 4 L / D$. The averaged Stanton number appearing in the formulation is $\overline{S t}=N u / R e \overline{P r}$ with $\overline{P r}=\bar{C}_{p} \mu / k$, where the averaged specific heat is $\bar{C}_{p}=\left(h_{w}-h\right) /\left(T_{w}-T\right)$, as in many heat transfer correlations proposed for supercritical pressure fluids.

Since the similarity requests that the trends of wall dimensionless enthalpy, $h_{w}^{*}$, and the one in bulk, $h^{*}$, are equal for the two fluids, this form of the Newton law suggests also that the product $\overline{S t} L / D$ should be nearly the same. This is a difficult requirement to be imposed exactly a priori because of the different properties of the fluids; however, satisfying it at least approximately turned out to be possible by choosing the fluid pressures in order to find sufficiently constant ratios of the averaged Stanton numbers for the two fluids in the considered range of the dimensionless enthalpy. Whenever this was sufficiently assured, compensating for the non-unity ratio of the Stanton numbers by changing the $L / D$ parameter was found to be practicable; recipes in this regard were discussed in reference [26] and applied to the selection of pressure for the four different fluids in relation to the experimental data by Watts [27] and Kline [28].

In summary, the following steps must be followed in applying the rationale:

1. define the corresponding pressures for the different fluids, according, for instance, to the recipe suggested in reference [26], i.e., trying to obtain sufficiently constant ratios between the Stanton number of the reference and simulant fluids as a function of the dimensionless enthalpy;

2. assume the same values of $N_{S P C}=-h_{i n}^{*}$ and $N_{T P C}$, to assign a similar trend of bulk dimensionless enthalpy;

3. impose the same values of the Froude and the Reynolds numbers at the channel inlet, thus adjusting the pipe diameter, as mentioned above;

4. guess the $L / D$ ratio on the basis of the formula $N_{T P C}=\overline{S t}\left(h_{w}^{*}-h^{*}\right) 4 L / D$;

5. perform CFD analyses for the new fluid, iterating on the heat flux and the on the $L / D$ ratio while keeping satisfied the relationship $N_{T P C}=\left[q^{\prime \prime} \beta_{p c} / G C_{p, p c}\right](4 L / D)$, until the trends of the wall dimensionless enthalpy as a function of the bulk dimensionless enthalpy are nearly the same for the two considered fluids; this process is required because of the approximate nature of any similarity theory, together with the high sensitivity of the phenomena to be predicted.

The above-described steps are represented in Figure 5, which reports relevant examples of plots illustrating the concepts addressed above. In particular, in STEP 1, the trend of the Prandtl or the Stanton number must be preliminarily looked at to get an idea of the possible "similar" pressures for the different fluids. In STEP 2, the required dimensionless enthalpy range must be imposed correctly for all the different fluids, because, as it can be noted in the related plot, it critically determines the expansion capability of the fluid. STEP 3 involves imposing the values of velocity at the inlet and of the diameter, while STEPS 4 and 5 require guessing and iterating on $q^{\prime \prime}$ and $L / D$ while performing RANS CFD analyses aimed at obtaining the targeted trend of $h_{w}^{*}$ as a function of the bulk dimensionless enthalpy. 


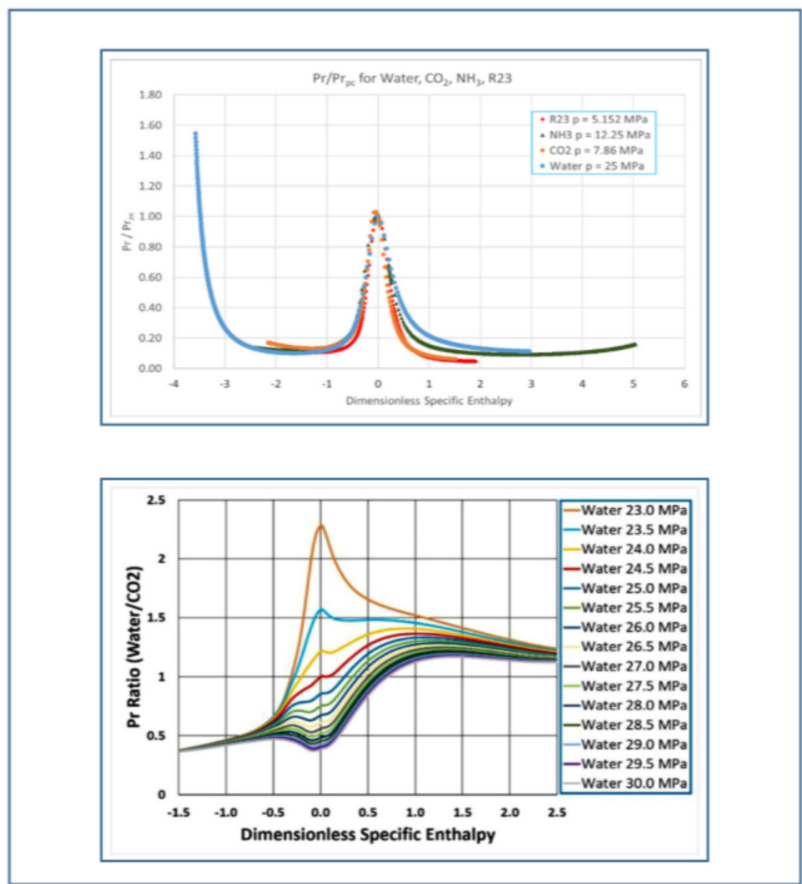

STEP 1: Select operating pressures, basing on inspection of the Pr and/or St numbers in the $h^{*}$ range

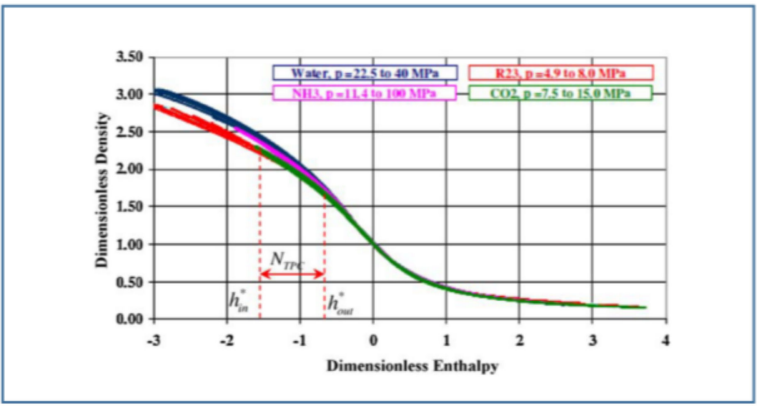

STEP 2: Impose the same $\mathbf{N}_{\text {SPC }}$ and $\mathbf{N}_{\text {TPC }}$ to assure the same expansion capability of the fluid in bulk

$\frac{w_{\text {in }, \text { fluid }-2}}{w_{\text {in }, \text { fluid }-1}}=\left[\frac{v_{\text {in }, \text { fluid }-2}}{v_{\text {in }, \text { fluid }-1}}\right]^{1 / 3}$
$\frac{D_{\text {fluid }-2}}{D_{\text {fluid }-1}}=\left[\frac{v_{\text {in }, \text { fluid }-2}}{v_{\text {in }, \text { fluid }-1}}\right]^{2 / 3}$

STEP 3: Impose the same Froude and Reynolds numbers at the inlet

Figure 5. Steps in the rationale of the similarity theory.

\begin{tabular}{|c|}
\hline$N_{\text {TPC }}=\overline{S t}\left(h_{w}^{*}-h^{*}\right) 4 L / D$ \\
STEP 4: Guess the L/D ratio
\end{tabular}

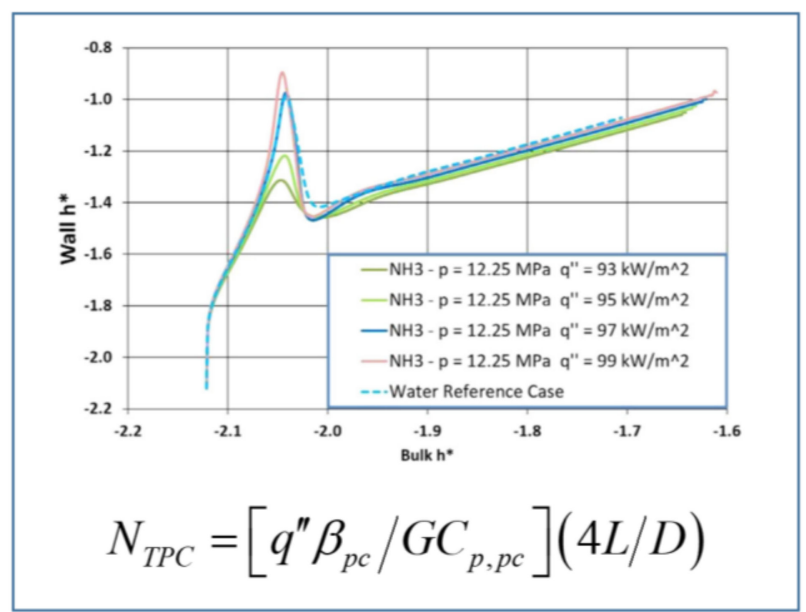

STEP 5: Iterate by RANS analyses on $q^{\prime \prime}$ and the $L / D$ ratio until convergence of the $h^{*}$ at the wall to the reference trend 
Though this rationale may look somehow complex, the authors applied it for some time without great difficulties and with good results. Some flexibility does exist in its application, e.g., in relation to the choice of the "similar" pressures and in the definition of the optimum heat flux to be obtained by iterations, so that the process is actually less cumbersome than it may look like.

A prerequisite for the reliability of the predictions is that the adopted reference experimental data must be acceptably well-predicted by the RANS CFD model. Whether it is not the case, it is not meant that the results of the analysis will not provide a good similarity, as was shown in reference [24] by the use of a two-equation turbulence model that was not particularly successful in predicting the reference data. In fact, wrong predictions in front of the experimental data may be useful anyway to find similarities among the predicted trends for different fluids, something mainly dependent on the fluid properties and not on the specific adopted turbulence model. However, it is quite obvious that a reliable planning of the counterpart experiments should start from some reliable prediction of the experimental data obtained with a specific fluid in order to safely postulate that the model is adequate to represent similar phenomena for another fluid.

\section{Overview of the Results Obtained So Far}

The work for setting up the present form of the similarity theory started long ago [13] but found a first consolidation with a recent $\mathrm{PhD}$ thesis [22]. The related conclusions were published in a journal paper [23], highlighting a procedure of application mostly identical to the one described above. However, only recently, the full capabilities of the theory were better recognised and found further confirmation in the application to both DNS and experimental data. It must be recognised that, in this regard, the availability of systematic experimental data both by Watts [27] and Kline [28], respectively with water and carbon dioxide, represented a key aspect in stimulating understanding and providing a confirmation of the trends that would be difficult to conceive without experiencing them in the excellent sequences of data obtained by varying the relevant parameters in the steps.

\subsection{Application to DNS Data}

A first recent paper [24] was published after receiving stimulus from a group of colleagues from the University of Sheffield, who published DNS analyses [29] based on the rough ideas of similarity presented in reference [13]. The surprising aspect in the results by the University of Sheffield was that the similarity rationale worked almost perfectly in its formulation of reference [13], which was found quite imperfect at the time by RANS calculations. In particular, the analysis showed that, in a flow duct with a relatively modest variation of the bulk dimensionless enthalpy, the curves of wall dimensionless enthalpy obtained by DNS calculations could merge almost in a single trend for four different fluids (Figure 6a). This result was explained in reference [24] with the short range of dimensionless enthalpy involved in the analyses and the selection of pressures that granted almost the same values of the Prandtl number across the pseudocritical threshold for the four fluids. This was confirmed by RANS analyses that reproduced similar data for the four fluids (Figure 6b). The accuracy in the similarity of the behaviour observed by the fluids suggested that the adopted rationale was very promising. 


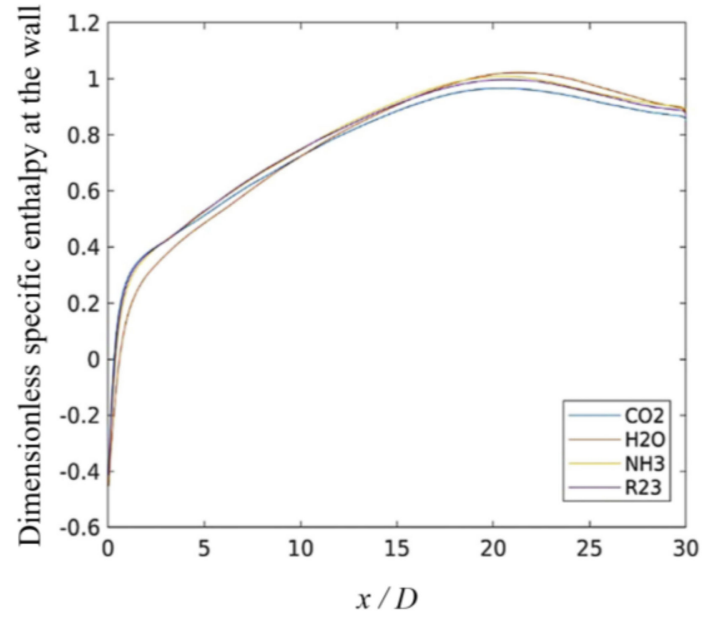

(a) DNS calculations by Sheffield University

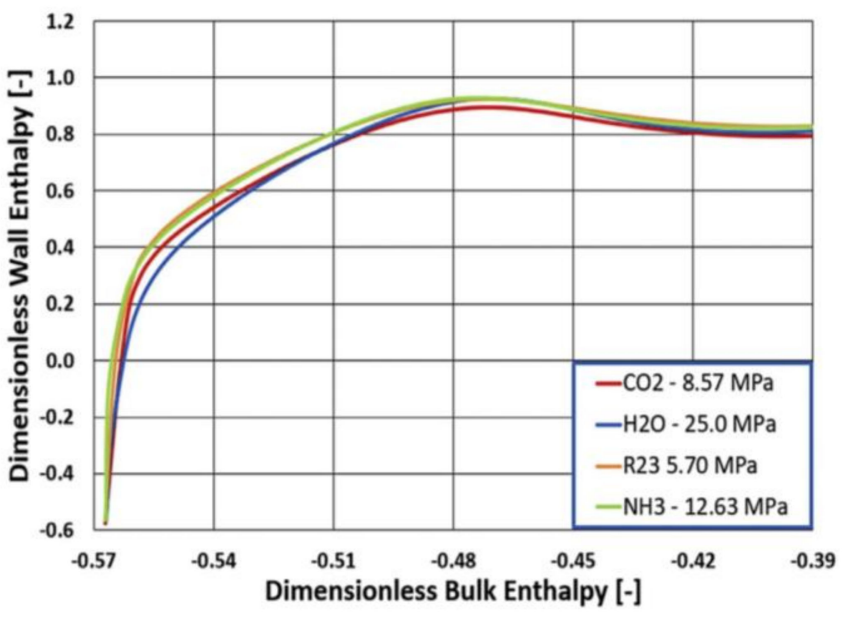

(b) RANS calculations

Figure 6. Results from the DNS and RANS calculations in a dimensionless form (adapted from reference [24]).

\subsection{Application to $\mathrm{CO}_{2}$ and Water Experimental Data}

As already mentioned, the carbon dioxide data by Kline [28] represented an excellent set of experiments exhibiting systematic changes in boundary conditions. This suggested useful information on the effect of operating conditions on the heat transfer regimes occurring along the flow duct, contributing to clarify some of the most debated issues, e.g., in relation to the onset of deterioration and on its characteristics after initiation. In particular, the interest of Kline's data is mainly due to the fact that they cover a full range of heat transfer phenomena, involving "normal/enhanced" heat transfer, "deterioration" and the final restoration of heat transfer at the transition to the gas-like phase. This was already discussed in Section 2 while presenting the features of heat transfer at supercritical pressure (see, e.g., Figure 3). The good predictions obtained by the Algebraic Heat Flux model $[19,20]$ previously implemented in the STAR-CCM + CFD code [21] provided confidence in the results obtained by the application of the similarity rationale, as described in Figure 5. This step in the evolution of the theory [25] had the following main results:

- the rationale applied in the work on DNS data [24] was extended to include strategies for dealing with situations in which the Prandtl numbers of the different fluids were largely different, suggesting recipes for achieving good results depending on the dimensionless enthalpy window addressed across the pseudocritical threshold;

- in particular, different strategies were used for Watts' water data [27] and Kline's carbon dioxide ones [28], obtaining preliminary results that confirmed the possibility to extend the similarity theory to more general cases in terms of the relative values of the Prandtl numbers.

Two works [26,32] made it possible to dig more deeply into the potential of the similarity theory by examining more complete sets of Kline's and Watts' data, thus providing greater information on the capabilities of the similarity theory. In particular, the trends of the relevant dimensionless numbers along the channel were represented, providing sufficient confidence that the similarity rationale worked as predicted by the theory.

It is interesting to note that the use of carbon dioxide data (by Kline) or water data (by Watts) as reference cases for the application of similarities could be considered to refer to two different points of view:

1. when basing on water data, the question to be answered is what could be the trend expected for simulant fluids (such as $\mathrm{CO}_{2}, \mathrm{NH}_{3}$ and $\mathrm{R} 23$ ) in "similar" conditions, i.e., how they would appear in experiments with those fluids exhibiting the same phenomena as those observed in water; this question represents a "direct" problem of scaling that an experimenter should solve for designing tests with a simulant fluid; 
2. when using carbon dioxide data, the addressed problem is analogous but, in the latter case, involves an "inverse" problem, i.e., to establish which phenomena in water could be expected as similar to those observed with the simulant fluids.

No matter what we may call "direct" or "inverse" problems, the high interest in solving both of them is clear, e.g., in the design phase of experimental apparatuses with simulant fluids or in translating the information gathered with several different fluids into similar information for water as the main fluid of interest for cooling nuclear reactors. This is why the two sets of data were both considered for the application of the similarity theory.

Figure 7 reports the original trends of some wall temperature data by Kline for carbon dioxide and the related predictions by the RANS calculations, compared with the trends obtained in similarity for the other three fluids: water, $\mathrm{NH}_{3}$ and $\mathrm{R} 23$. On the other hand, the plots in Figure 8 report the trends along the channel axis of the relevant dimensionless numbers for the four fluids.

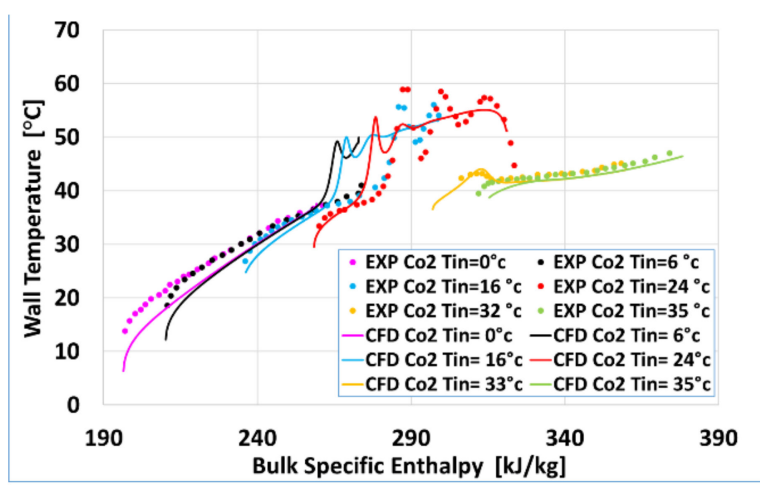

(a) $\mathrm{CO}_{2}$ data and their prediction

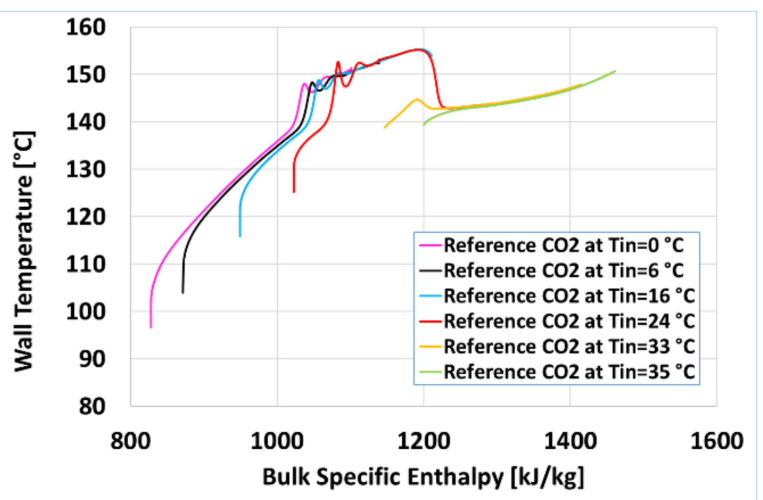

(c) similar trends for $\mathrm{NH}_{3}$ at $12.5 \mathrm{MPa}$

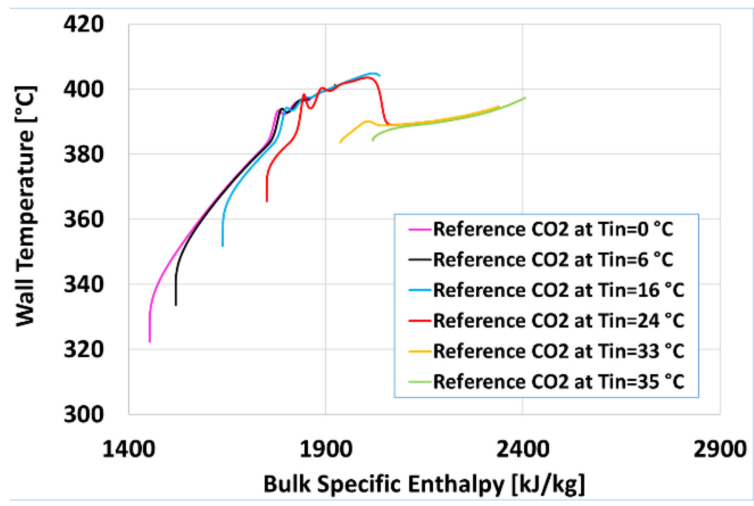

(b) similar trends for water at $24.5 \mathrm{MPa}$

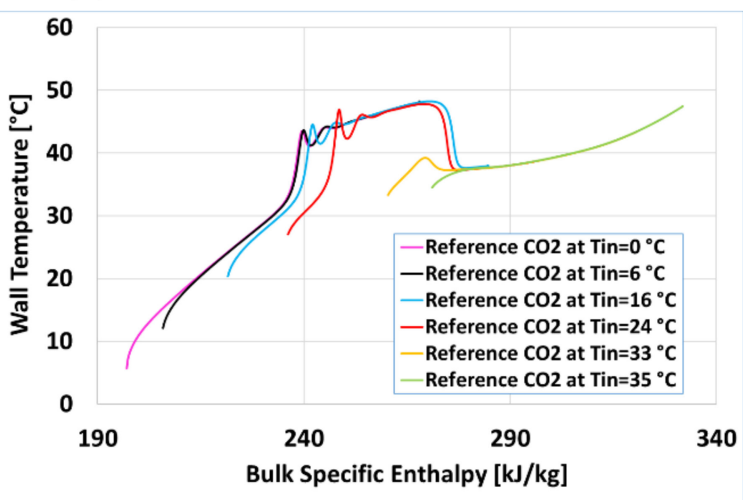

(d) similar trends for $\mathrm{R} 23$ at $5.56 \mathrm{MPa}$

Figure 7. Prediction of Kline's $\mathrm{CO}_{2}$ data at $\mathrm{q}^{\prime \prime}=18 \mathrm{~kW} / \mathrm{m}^{2}$, and trends obtained by the application of the similarity theory for water, $\mathrm{NH}_{3}$ and $\mathrm{R} 23$.

This information can be summarised as follows.

- The plots in Figure 7 show that the present similarity theory is able to translate the relevant qualitative information about the different heat transfer regimes from the case of one fluid to the other. Indeed, very similar phenomena related to normal or enhanced heat transfer, onset of deterioration, repeated wall temperature oscillations and the restoration of heat transfer efficiency at the transition to the gas-like region are very well-represented, with all the fluids in strict similarity. This set of pictures is one of the most meaningful ways to show the effectiveness of the similarity rationale, since it shows that different fluids at different pressures are predicted to behave in strict phenomenological accordance. 
- A more quantitative perspective is presented in the plots of Figure 8, reporting one of the addressed cases by Kline and its three counterparts for water, ammonia and R23 in terms of the dimensionless quantities. The following comments apply.

- The matching of the trends of dimensionless enthalpy at the wall (Figure 8a) is actually the main target pursued by the similarity rationale, i.e., the one which is sought for in iterations on heat flux for declaring convergence; this represents the basic achievement supporting all the other aspects of the similarity; in considering the obtained trends, it is necessary to remember that a similar distribution of dimensionless enthalpy means a similar distribution of fluid density, with all the related consequences in terms of heat transfer regimes, as discussed in Section 2.

$\checkmark$ The trends of the Richardson and of the Froude numbers are then predicted in close similarity (Figure $8 b, c$ ) as a consequence of preserving the same $N_{S P C}$ and $N_{T P C}$, assuring the same expansion (i.e., acceleration) of the fluid in bulk, and because of the matching observed in the dimensionless enthalpy at the wall.

- The Nusselt number cannot be exactly the same for the different fluids (Figure 8d), since it is instead the product $\overline{S t} L / D$ to be preserved (Figure $8 \mathrm{~g}$ ); indeed, the differences in the local values of the Reynolds and the Prandtl numbers do generate different values of the Nusselt number.

$\checkmark$ On the other hand, at least for this set of data, it is remarkable to consider that a very good matching occurs between the values of the ratio of the Nusselt number to the one calculated by the Dittus-Boelter correlation (named Nu_DB in Figure 8e); this could suggest that the Dittus-Boelter correlation could be taken as the basis, then be corrected as a function of the other dimensionless numbers in order to achieve a general correlation applicable to different fluids able to predict heat transfer deterioration; this assumption, which is actually at the basis of many of the known correlations for supercritical pressure fluids, must be confirmed by elaborating the available experimental data.

$\bigcirc \quad$ Though preserved at the inlet, the Reynolds number evolves differently for the different fluids (Figure 8f), suggesting that it is possible only to keep some rough similarities in the turbulence levels along the heated pipe.

These results confirm, to a large extent, the assumptions at the basis of the similarity theory. Further data were obtained by a thorough analysis of Watts' data [27], obtained with water at $25 \mathrm{MPa}$, recently performed in the frame of a MSc thesis [38], whose details will appear in a forthcoming paper [32].

Typical results from that work are reported in Figures 9-12. After the usual check about the correctness on the reasonable prediction by the CFD model of the experimental data (Figure 9), the similarity rationale was applied, obtaining corresponding results for the different fluids. A sample of the obtained results are shown in Figures 10-12 for increasing levels of the flow rate, which causes decreasing levels of heat transfer deterioration. As it can be noted, while the agreement between the trends of water and ammonia is quite good, $\mathrm{CO}_{2}$ and $\mathrm{R} 23$ show an oscillating behaviour in the left part of the curves, which can be interpreted as cycles of deterioration and restoration, though the average trend of the curves is approximately the same. At the moment, it cannot be understood if this behaviour is an artefact of the adopted RANS model (e.g., failing in predicting the right trend for wall dimensionless enthalpy) or if this cycling of wall temperatures can be really expected in the case of these fluids, e.g., because of their higher Prandtl number. 


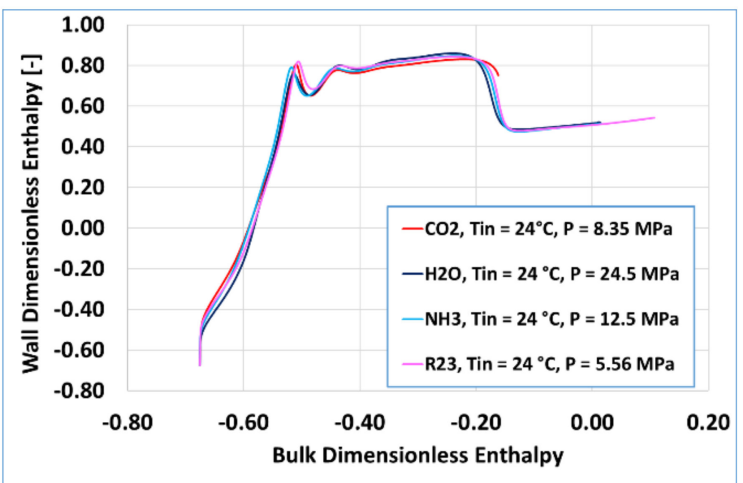

(a) wall dimensionless enthalpy

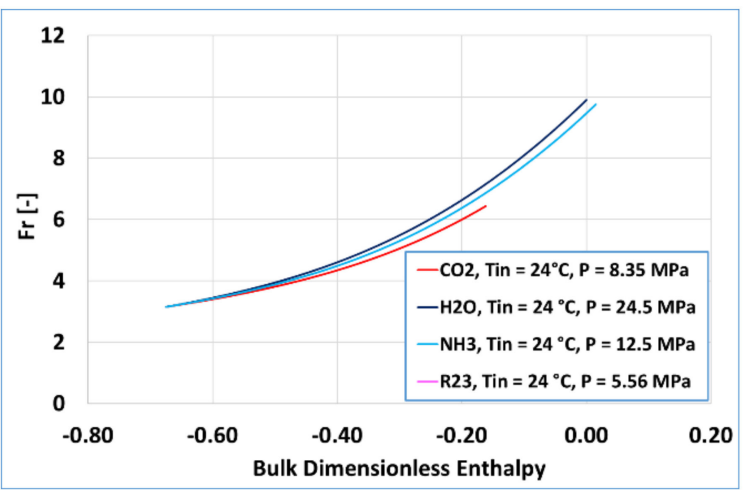

(c) Froude number

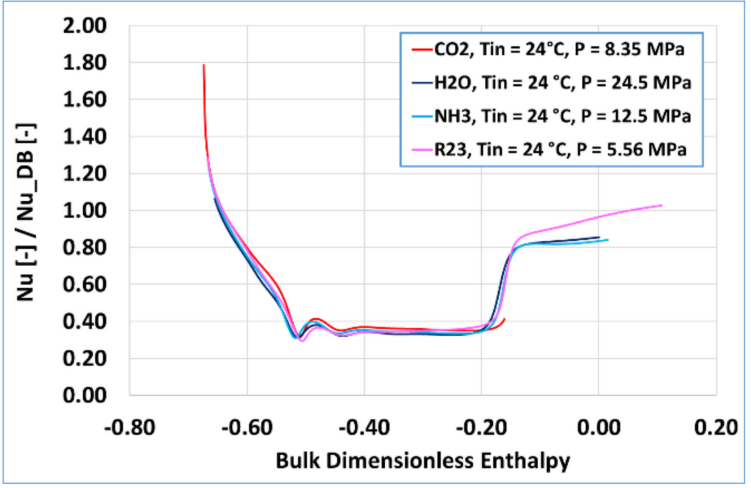

(e) Nusselt number ratio

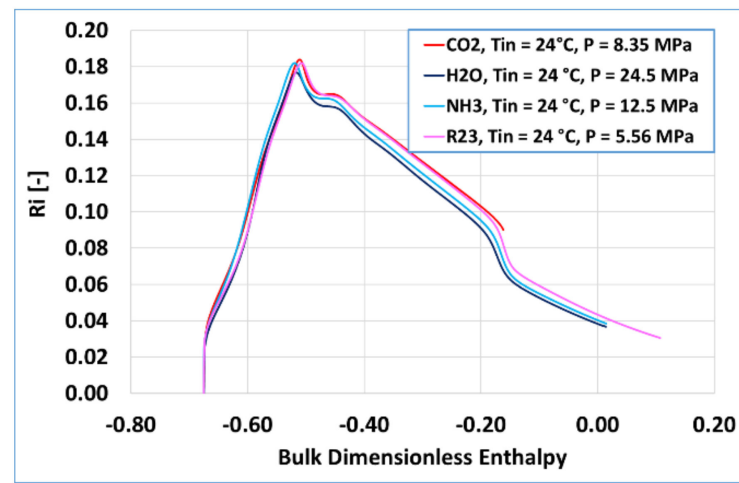

(b) Richardson number

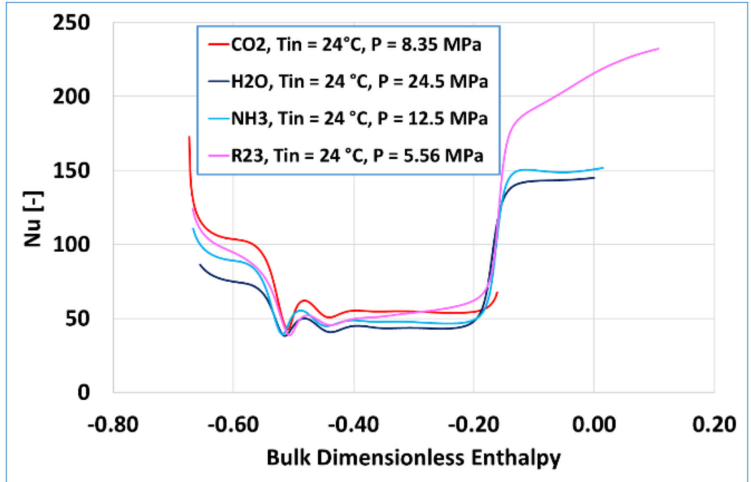

(d) Nusselt number

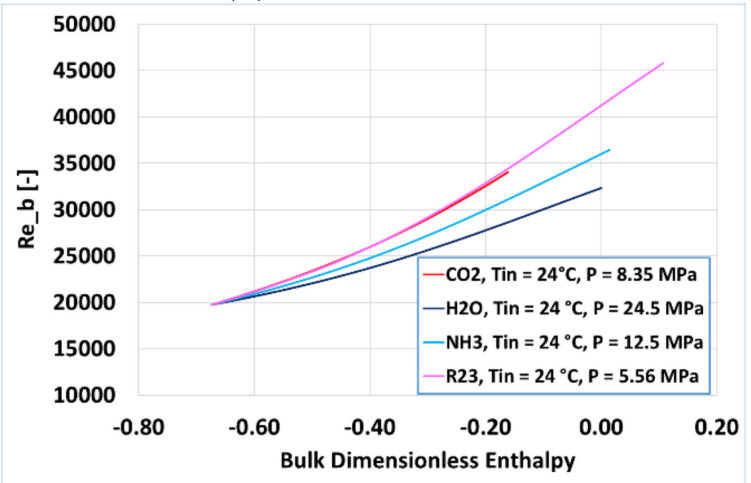

(f) Reynolds number

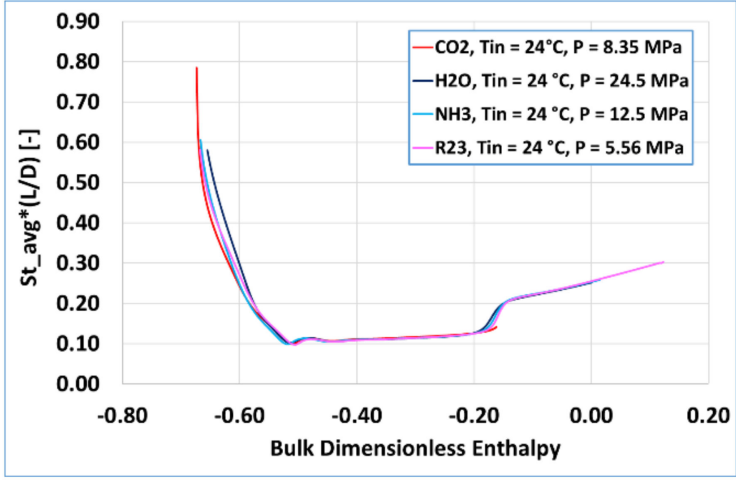

(g) $\overline{S t} L / D$

Figure 8. Trends of the relevant dimensionless numbers along the channel showing similarity for the case of Kline's data with $\mathrm{q}^{\prime \prime}=18 \mathrm{~kW} / \mathrm{m}^{2}$ and $\mathrm{T}_{\mathrm{in}}=24^{\circ} \mathrm{C}$. 


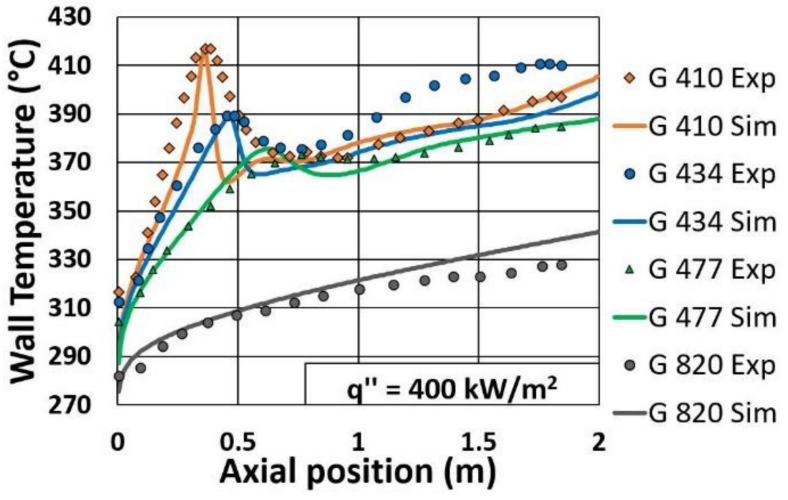

Figure 9. Selected CFD results for Watts' data at $\mathrm{T}_{\mathrm{in}}=250^{\circ} \mathrm{C}$ and $400 \mathrm{~kW} / \mathrm{m}^{2}$.

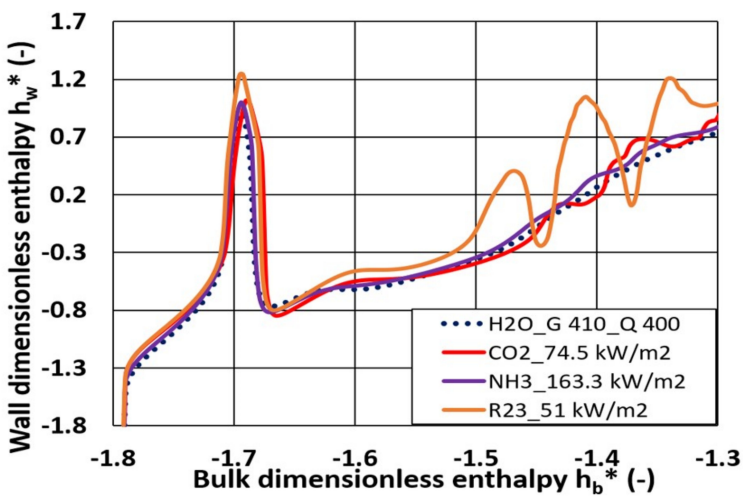

Figure 10. Predictions with the best heat fluxes for Watts' data (Reference Case: $G=410 \mathrm{~kg} / \mathrm{m}^{2} \mathrm{~s}$ ).

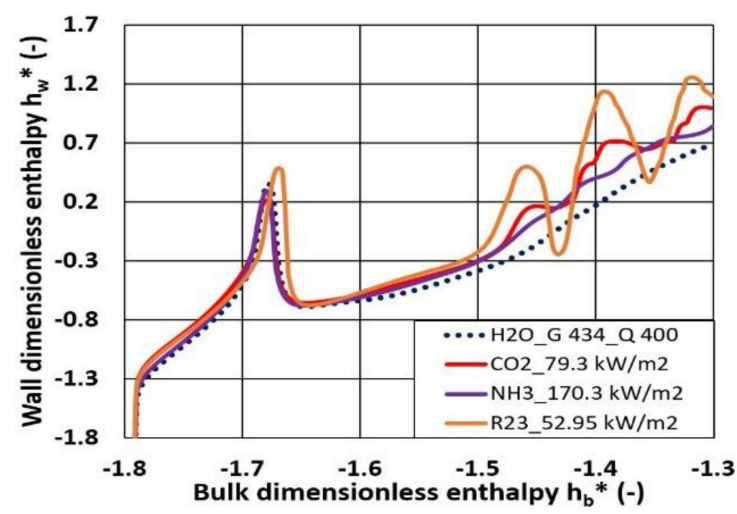

Figure 11. Predictions with the best heat fluxes for Watts' data (Reference Case: $G=434 \mathrm{~kg} / \mathrm{m}^{2} \mathrm{~s}$ ).

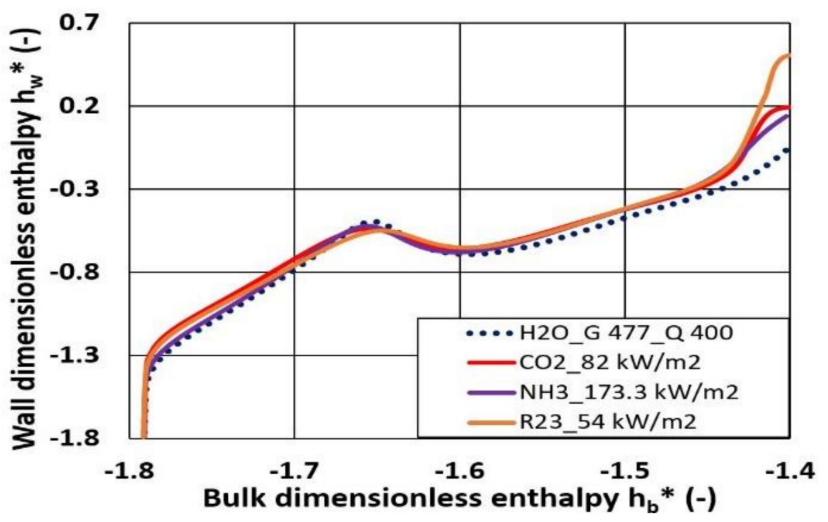

Figure 12. Predictions with the best heat fluxes for Watts' data (Reference Case: $G=477 \mathrm{~kg} / \mathrm{m}^{2} \mathrm{~s}$ ). 


\subsection{Extension to Non-Uniform Axial Heat Flux Distributions}

Recently a further advancement was carried out [31] to assess the applicability of the similarity theory in the case of nonuniform distributions of linear power along the fuel rods. This step was necessary because of the well-known nonuniform production of power along the axis of a nuclear reactor, which is sometimes also simulated in experimental facilities. Since the similarity theory was developed for the uniform linear power, it was necessary to firstly theoretically assess the feasibility of this extension and then proceed to verify the developed assumptions by RANS calculations.

The reader is referred to a specific publication [31] for the supporting analytical developments. Some of the results obtained with a sinusoidal power distribution, starting from some of the boundary conditions of the experimental data by Kline [28] with carbon dioxide (except for the power distribution), are illustrated herein for the case of water. In practical terms, after checking for a good prediction in reproducing the corresponding experimental case with uniform power in the dataset by Kline, a calculation was run with $\mathrm{CO}_{2}$, with the sinusoidal power distribution having the same average value. The results of this calculation were then assumed as a target trend of dimensionless enthalpy at the wall to be obtained in similarity with water and other fluids.

Figures 13 and 14, in particular, show the trends of wall dimensionless-specific enthalpy for water as a function of dimensionless bulk enthalpy at different attempted average heat fluxes, according to the iteration process described in STEP 5 of the methodology in Figure 5. Figures 15 and 16 report the same data as a function of the axial coordinate along the pipe, clearly showing the need of changing the $L / D$ ratio according to the presented rationale. This aspect is described by Figure 17, reporting the heat flux distributions that should be used with $\mathrm{R} 23$, water, $\mathrm{NH}_{3}$ and $\mathrm{R} 134 \mathrm{a}$ in order to get a reasonable similarity with respect to the reference cases considered with $\mathrm{CO}_{2}$. Though this characteristic of the rationale can be found displeasing for experiments, since it will oblige to control the shape of the heating power in dependence of the fluid, it is believed that, in some cases, it may be accomplished by the computerised control of locally distributed heaters. Nevertheless, this feature represents an inescapable outcome of the theory; though the changes in $L / D$ are generally modest, they cannot be completely ruled out.

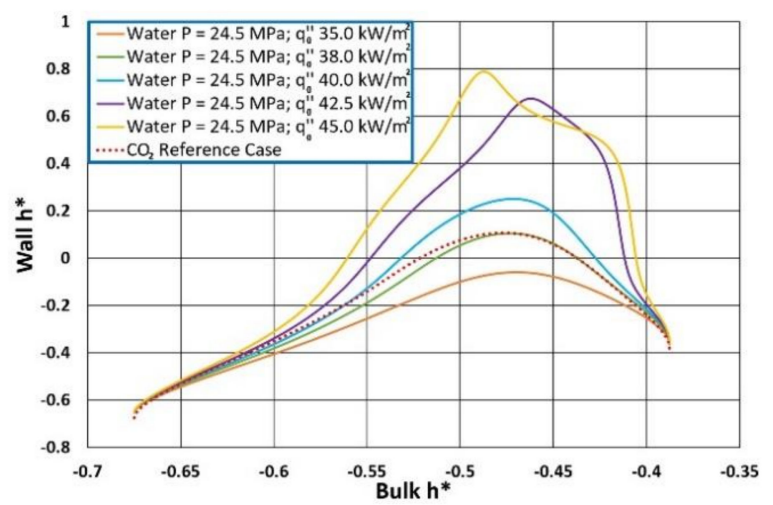

Figure 13. Wall $\mathrm{h}^{*}$ trends for water at $24.50 \mathrm{MPa}$ for a reference case with $\mathrm{CO}_{2}, \mathrm{q}_{0}{ }^{\prime \prime}=10 \mathrm{~kW} / \mathrm{m}^{2}$ and $\mathrm{T}_{\text {in }}=24^{\circ} \mathrm{C}$. 


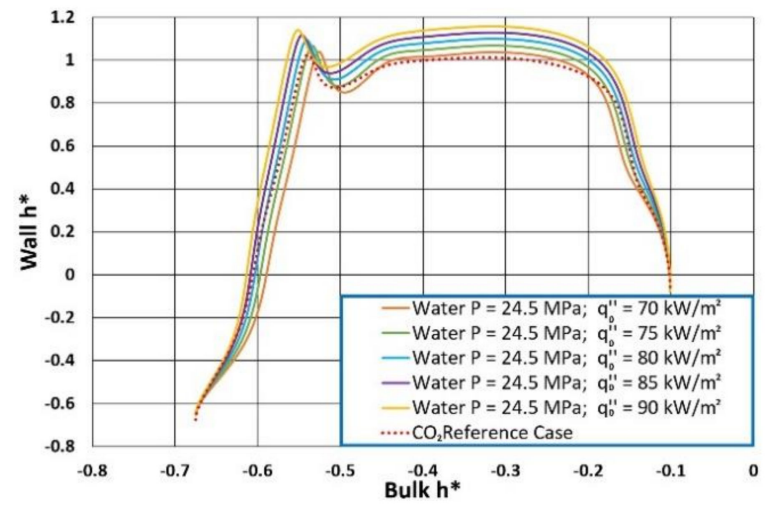

Figure 14. Wall $\mathrm{h}^{*}$ trends for water at $24.50 \mathrm{MPa}$ for a reference case with $\mathrm{CO}_{2}, \mathrm{q}_{0}{ }^{\prime \prime}=20 \mathrm{~kW} / \mathrm{m}^{2}$ and $\mathrm{T}_{\text {in }}=24^{\circ} \mathrm{C}$.

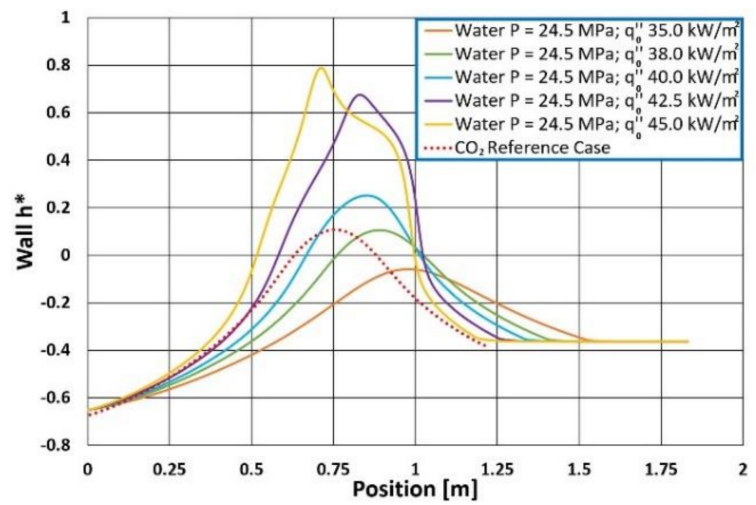

Figure 15. Wall $\mathrm{h}^{*}$ trends for water at $24.50 \mathrm{MPa}$ as a function of the axial position for a reference case with $\mathrm{CO}_{2}, \mathrm{q}_{0}^{\prime \prime}=10 \mathrm{~kW} / \mathrm{m}^{2}$ and $\mathrm{T}_{\text {in }}=24^{\circ} \mathrm{C}$.

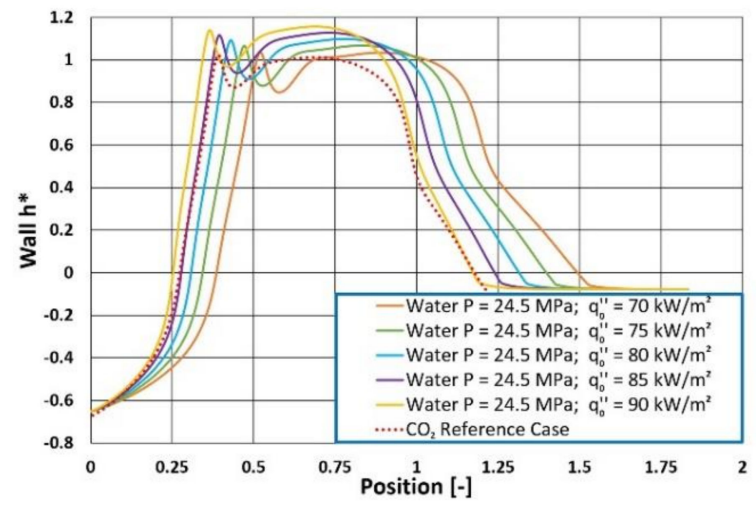

Figure 16. Wall $\mathrm{h}^{*}$ trends for water at $24.50 \mathrm{MPa}$ as a function of the axial position for a reference case with $\mathrm{CO}_{2}, \mathrm{q}_{0}^{\prime \prime}=20 \mathrm{~kW} / \mathrm{m}^{2}$ and $\mathrm{T}_{\text {in }}=24^{\circ} \mathrm{C}$. 


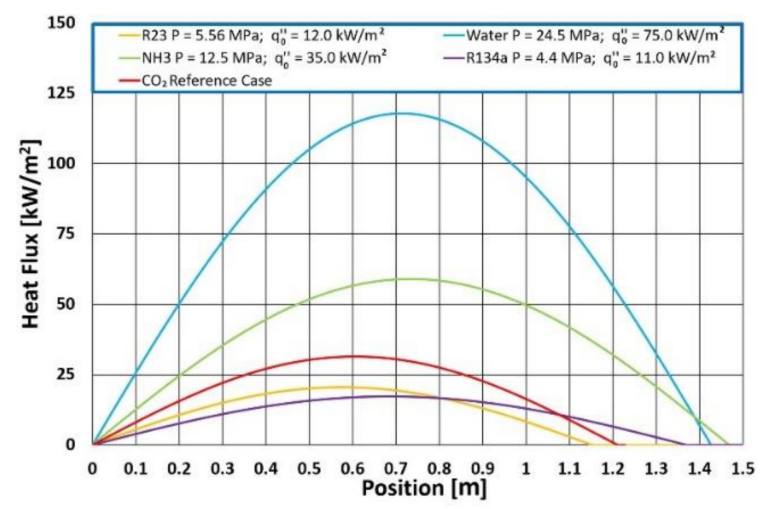

Figure 17. Heat flux distributions for the best-matching operating conditions for the reference $\mathrm{CO}_{2}$ case considering $\mathrm{q}_{0}^{\prime \prime}=20 \mathrm{~kW} / \mathrm{m}^{2}$ and $\mathrm{T}_{\text {in }}=24^{\circ} \mathrm{C}$.

\subsection{Applications of Fluids Not Considered Before}

In the frame of the EU ECC-SMART Project (https:/ / ecc-smart.eu/), experiments making use of R134a will be performed. Since the authors are partners in the Project Consortium and are involved in the analyses, it was considered appropriate to check how the similarity theory, applied mostly to fluids other than R134a, would have behaved in this case. Worries about the applicability of the theory were raised by an inspection of the trend of dimensionless density as a function of dimensionless enthalpy that, as discussed in previous sections, is at the basis of the close similarity in the behaviour of different fluids. In fact, considering Figure 18, it can be noted that R134a deviates from the behaviour of water and even from the one of $\mathrm{CO}_{2}$, quite consistently; in particular, R134a tends to have a lower expansion as a function of heating than water and carbon dioxide, something that may introduce distortions into the similarities.

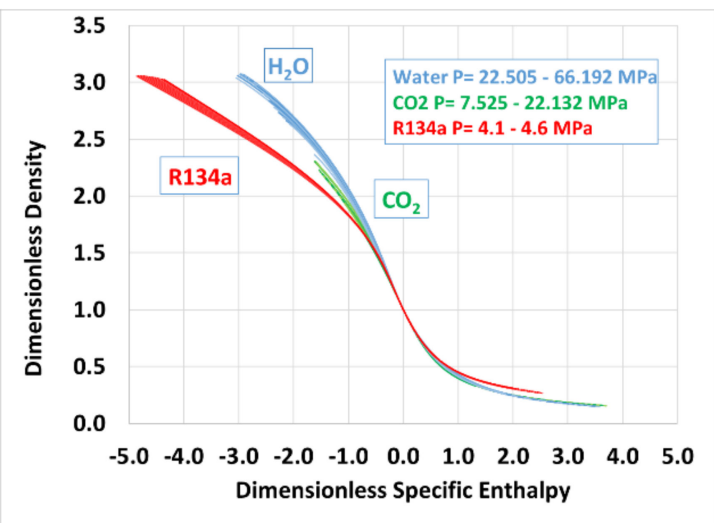

Figure 18. Dimensionless trend of density vs. specific enthalpy for R134a in comparison with water and $\mathrm{CO}_{2}$.

In order to take care of this aspect, a possible correction of the theory was envisaged, consisting of enlarging the effective dimensionless enthalpy range related to the new fluid in comparison with the others in order to bring the curves closer, thus obtaining similar expansion capabilities. This would have meant making use of different values of $N_{S P C}$ and $N_{T P C}$ in the application of the similarity for R134a in order to compensate for this lower tendency of the fluid to expand as a consequence of heating. Actually, the application of the usual rationale also in this case showed that this correction was not necessary, suggesting the presence of enough flexibility in the described steps of the similarity, so that satisfactory results could be achieved anyway.

Figure 19 reports the results of the calculations performed by the RANS model in the application of the similarity theory. As it can be noted, after checking the correctness 
of the prediction of the reference data by Kline (Figure 19a), we tried to obtain similar trends with R134a, with quite reasonable success (Figure 19b). In particular, the same heat transfer phenomena were exhibited with only slight phenomenological deviations; the similarity obtained seemed quite reasonable. Of course, the chain of similarities between $\mathrm{CO}_{2}$ and water and between $\mathrm{CO}_{2}$ and $\mathrm{R} 134 \mathrm{a}$ justifies the use of the latter to represent water conditions, which is a rather important achieved result.

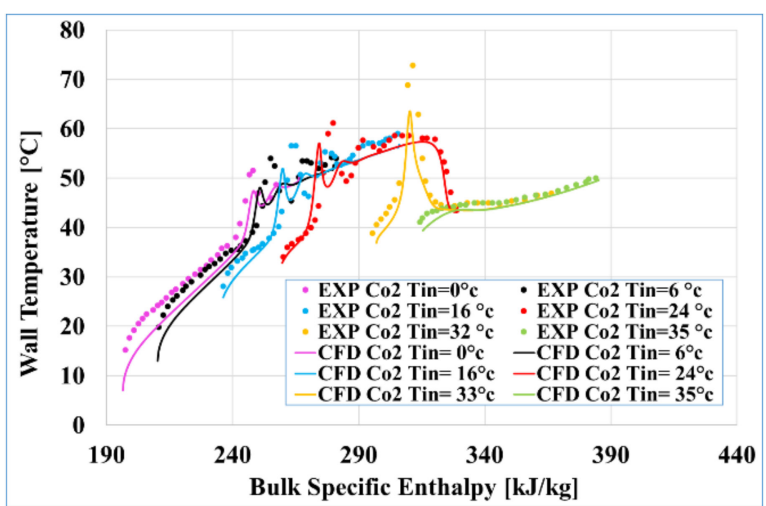

(a) experimental data and RANS predictions

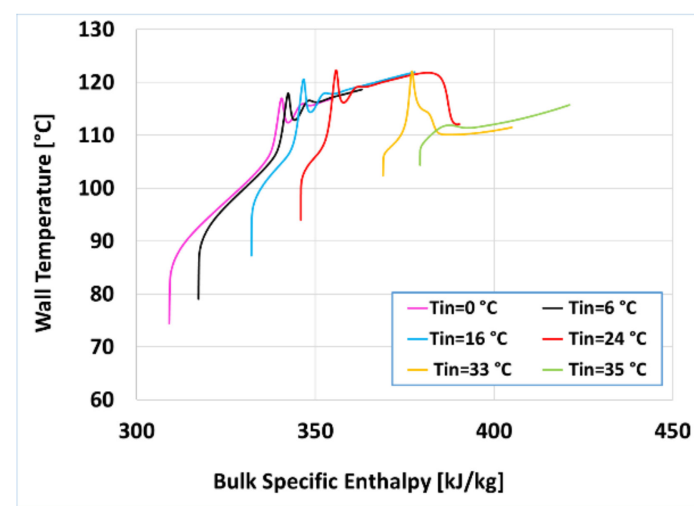

(b) RANS calculations for R134a

Figure 19. Trends of the wall temperature along the channel for the reference cases by Kline at $\mathrm{q}^{\prime \prime}=20 \mathrm{~kW} / \mathrm{m}$ compared with the trends of the wall temperature along the channel. RANS calculations with R134a at 4.47 MPa for the reference cases by Kline at $\mathrm{q}^{\prime \prime}=20 \mathrm{~kW} / \mathrm{m}$.

\subsection{Applicability of Some $\mathrm{CO}_{2}$ Experimental Data to SCWR Conditions}

Since, in previous analyses, carbon dioxide data by Kline were compared to water at $24.5 \mathrm{MPa}$ as the most appropriate pressure identified by the rationale for scaling with $\mathrm{CO}_{2}$ at $8.35 \mathrm{MPa}$, the need was felt to address also the most important pressure level of $25 \mathrm{MPa}$, being the rated pressure for many SCWR design concepts. While the full body of results of this elaboration will be published in a forthcoming conference paper, Figure 20 reports the predictions by the adopted RANS model of the water conditions inspired by the experimental data by Kline for a heating flux of $18 \mathrm{~kW} / \mathrm{m}^{2}$, already shown in Figure 7a. It is remarked, in fact, that the observation of an overall correctness of the phenomena prediction by the CFD model is the prerequisite for basing the predictions of similar conditions on them. As it can be noted from Figure 20, water at $25 \mathrm{MPa}$ is expected to behave in close similarity from a phenomenological point of view, with very similar trends as the ones observed and predicted for $\mathrm{CO}_{2}$. It is worth noting that the capability to "translate" information obtained by simulant fluids to the scale of postulated reactor conditions is very attractive for widening the database applicable to nuclear reactor conditions. With the use of the present similarity theory, it is now possible to make efficient use of a vast body of experiments obtained with different fluids, getting a clear idea of how water reactor conditions would be in close similarity. The obtained results also indicate some degree of tolerance in the application of the theory in relation to the choice of pressure; it clearly appears that though $24.5 \mathrm{MPa}$ was considered an ideal value for the water pressure, $25 \mathrm{MPa}$ is nevertheless still adequate to provide a picture of the phenomena in a close similarity. 


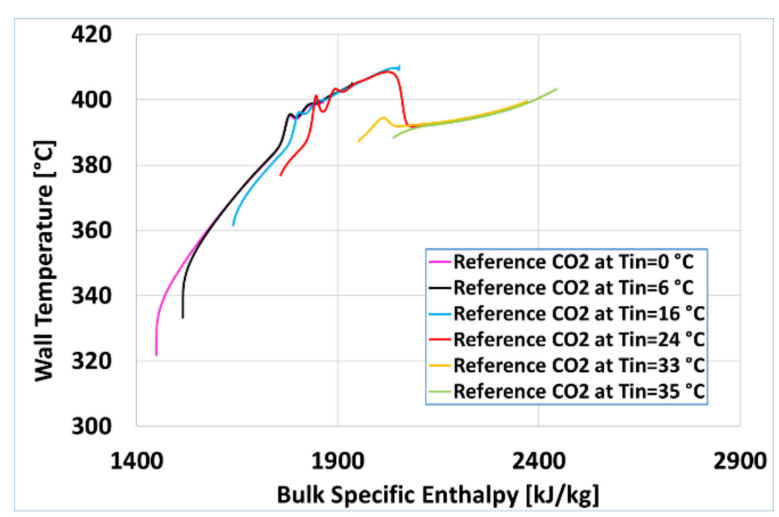

Figure 20. Trends of the wall temperature along the channel. RANS calculations with water at 25 MPa for the reference cases by Kline at $\mathrm{q}^{\prime \prime}=18 \mathrm{~kW} / \mathrm{m}^{2}$.

\section{Conclusions}

The results shown in this paper support the correctness of the assumptions at the basis of a similarity theory, whose basic ideas were proposed long ago [13], anyway requiring a sound correction and refinement that was achieved only in recent steps [22-26]. The validity of the theory is presently supported by RANS calculations, which allowed describing the promising features of the proposed rationale in the application in terms of the different fluids and boundary conditions.

This rationale can be now used to plan experimental tests that may provide further confirmation and assessment of this theory. It must be remarked that, among the different theories proposed so far for fluid-to-fluid similarity in heat transfer to supercritical pressure fluids, the present one was developed basing on consistency with the experimental observations of predicted trends by RANS calculations. Moreover, its principles were devised considering the overwhelming importance that the changes in fluid density have on the observed heat transfer regimes, with regards to the challenging aspects of deterioration.

The usefulness of this theory is clearly related to the capability to unify the experimental information obtained by very different fluids, thus providing an enlargement of the database to be used for model and correlation assessment related to water conditions. Considering the present state-of-the-art in the field of empirical correlations for predicting heat transfer deterioration phenomena, the present theory provides hope that a better understanding of the key phenomena and of the relevant dimensionless numbers it involves may provide decisive developments. The present work by the authors focused on revising the existing heat transfer correlations and in understanding how they can be reconsidered in view of the results of the similarity theory. These developments, having a general interest, will be also a sound contribution to the ongoing efforts for advancing the predictive capabilities required for the licensing of nuclear reactors cooled and moderated by water at supercritical pressure.

Author Contributions: Conceptualization, A.P. and W.A.; methodology, A.P.; software, A.P.; validation, S.K., A.P.; formal analysis, all authors.; investigation, S.K. and A.P.; writing-original draft preparation, W.A.; writing—review and editing, all authors.; funding acquisition, W.A. All authors have read and agreed to the published version of the manuscript.

Funding: The latest applications of the theory reported in this paper were performed in the frame of the EU ECC-SMART project: this project received funding from the Euratom research and training programme 2019-2020 under grant agreement no. 945234.

Acknowledgments: The similarity theory was developed over the timespan of more than a decade, the latest developments occurring in the first months of 2020 during the first wave of the COVID-19 pandemic. The IAEA is gratefully acknowledged for the involvement granted in two subsequent CRPs on the SCWR thermal hydraulics in the past years.

Conflicts of Interest: The authors declare no conflict of interest. 


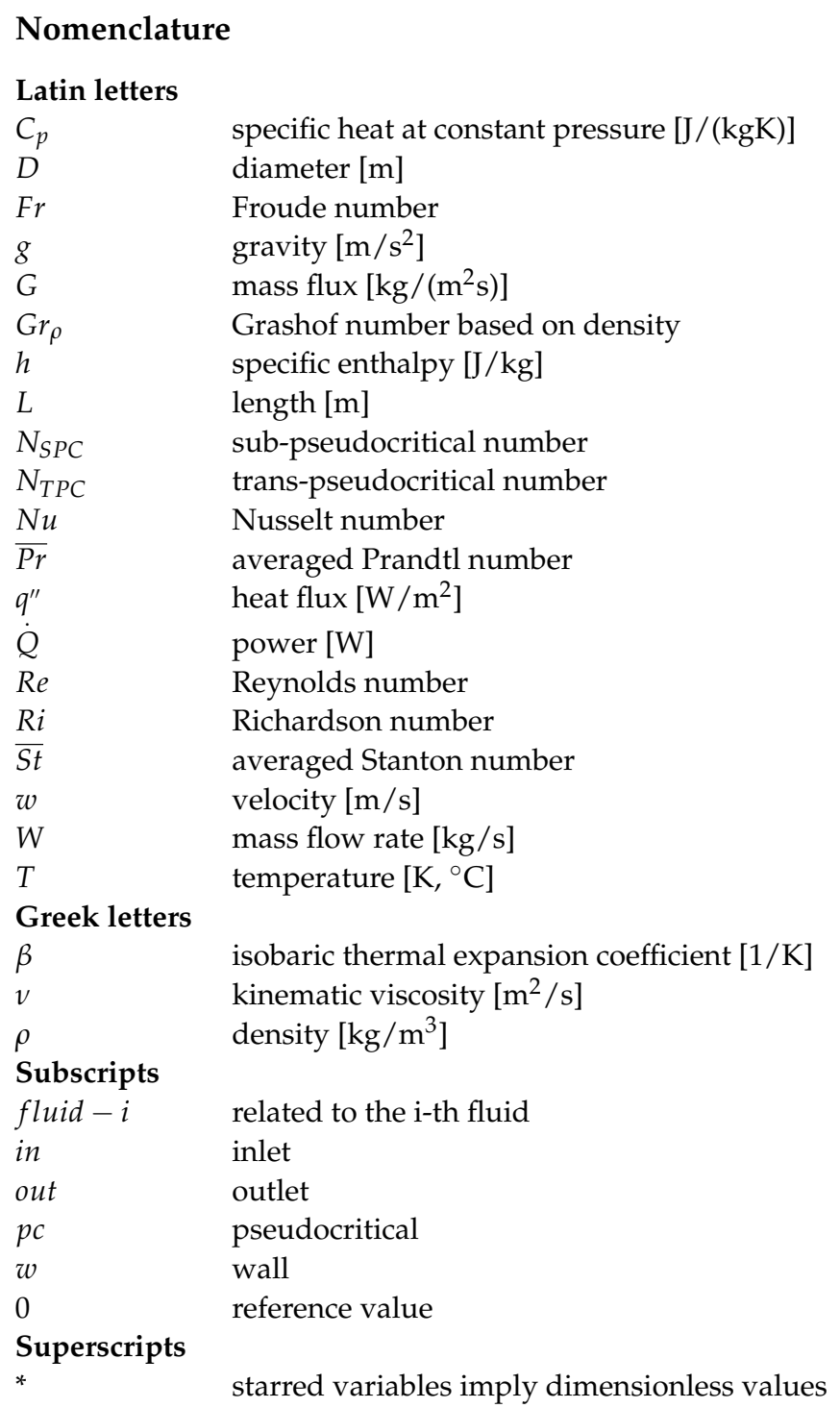

\section{References}

1. Jackson, J.; Hall, W. Influences of buoyancy on heat transfer to fluids flowing in vertical tubes under turbulent conditions. Turbul. Forced Convect. Channels Bundles 1979, 2, 613-640.

2. Licht, J.; Anderson, M.; Corradini, M. Heat transfer to water at supercritical pressures in a circular and square annular flow geometry. Int. J. Heat Fluid Flow 2008, 29, 156-166. [CrossRef]

3. Pioro, I.L.; Duffey, R.B. Heat Transfer and Hydraulic Resistance at Supercritical Pressure in Power Engineering Applications; ASME Press: New York, NY, USA, 2007.

4. $\quad$ Oka, Y.; Koshizuka, S.; Ishiwatari, Y.; Yamaji, A. Super Light Water Reactors and Super Fast Reactors: Supercritical-Pressure Light Water Cooled Reactors; Springer: New York, NY, USA, 2010.

5. Schulenberg, T.; Starflinger, J. High Performance Light Water Reactor: Design and Analyses; KIT Scientific Publishing: Karlsruhe, Germany, 2012.

6. Cheng, X.; Schulenberg, T. Heat Transfer at Supercritical Pressures-Literature Review and Application to an HPLWR; FZKA: Karlsruhe, Germany, 2001; Volume 6609.

7. International Atomic Energy Agency. Heat Transfer Behaviour and Thermohydraulics Code Testing for Supercritical Water Cooled Reactors (SCWRs); IAEA-TECDOC-1746; IAEA: Vienna, Austria, 2014.

8. International Atomic Energy Agency. Status of Research and Technology Development for Supercritical Water-Cooled Reactors; IAEATECDOC-1869; IAEA: Vienna, Austria, 2019.

9. International Atomic Energy Agency. Understanding and Prediction of Thermohydraulic Phenomena Relevant to Supercritical Water Cooled Reactors (SCWRs); IAEA-TECDOC-1900; IAEA: Vienna, Austria, 2020. 
10. Azih, C.; Yaras, M.I. Similarity Criteria for Modeling Mixed-Convection Heat Transfer in Ducted Flows of Supercritical Fluids. J. Heat Transf. 2017, 139, 122501. [CrossRef]

11. Mouslim, A.; Tavoularis, S. Direct tests of fluid-to-fluid scaling expressions for supercritical heat transfer in tubes. Int. J. Heat Mass Transf. 2020, 147, 118862. [CrossRef]

12. Ambrosini, W.; Sharabi, M. Dimensionless parameters in stability analysis of heated channels with fluids at supercritical pressures. Nucl. Eng. Des. 2008, 238, 1917-1929. [CrossRef]

13. Ambrosini, W. Discussion of similarity principles for fluid-to-fluid scaling of heat transfer behaviour at supercritical pressures. Nucl. Eng. Des. 2011, 241, 5149-5173. [CrossRef]

14. Sharabi, M.B.; Ambrosini, W.; Forgione, N.; He, S. Prediction of Experimental Data on Heat Transfer to Supercritical Water with Two-Equation Turbulence Models. In Proceedings of the Third International Symposium on SCWR-Design and Technology, Shanghai, China, 12-15 March 2007.

15. Sharabi, M.; Ambrosini, W.; He, S.; Jackson, J.D. Prediction of turbulent convective heat transfer to a fluid at supercritical pressure in square and triangular channels. Ann. Nucl. Energy 2008, 35, 993-1005. [CrossRef]

16. Pucciarelli, A.; Sharabi, M.; Ambrosini, W. Prediction of heat transfer to supercritical fluids by the use of Algebraic Heat Flux Models. Nucl. Eng. Design 2016, 297, 257-266. [CrossRef]

17. Papp, V.; Pucciarelli, A.; Sharabi, M.; Ambrosini, W. Use of Algebraic Heat Flux Models to Improve Heat Transfer Predictions for Supercritical Pressure Fluids. In Proceedings of the 24th 2016 International Conference on Nuclear Engineering, ICONE, Charlotte, NC, USA, 26-30 June 2016. V004T10A012.

18. Pucciarelli, A.; Ambrosini, W. Improvements in the prediction of heat transfer to supercritical pressure fluids by the use of algebraic heat flux models. Ann. Nucl. Energy 2017, 99, 58-67. [CrossRef]

19. Pucciarelli, A.; Ambrosini, W. Use of AHFM for simulating heat transfer to supercritical fluids: Application to carbon dioxide data. Int. J. Heat Mass Transf. 2018, 127, 1138-1146. [CrossRef]

20. Buzzi, F.; Pucciarelli, A.; Ambrosini, W. On the mechanism of final heat transfer restoration at the transition to gas-like fluid at supercritical pressure: A description by CFD analyses. Nucl. Eng. Des. 2019, 355, 110345. [CrossRef]

21. Simcenter STAR-CCM ${ }^{+}$, version 13.06; Documentation; Siemens PLM Software: Plano, TX, USA, 2018.

22. Pucciarelli, A. Analysis of Fluiddynamic and Heat Transfer Phenomena with Supercritical Fluids; Università di Pisa, Dipartimento di Ingegneria Civile e Industriale, Corso di Dottorato in Ingegneria Industriale: Pisa, Italy, 2017.

23. Pucciarelli, A.; Ambrosini, W. Fluid-to-fluid scaling of heat transfer phenomena with supercritical pressure fluids: Results from RANS analyses. Ann. Nucl. Energy 2016, 92, 21-35. [CrossRef]

24. Pucciarelli, A.; He, S.; Ambrosini, W. A successful local fluid-to-fluid similarity theory for heat transfer to supercritical pressure fluids: Merits and limitations. Int. J. Heat Mass Transf. 2020, 157, 119754. [CrossRef]

25. Pucciarelli, A.; Ambrosini, W. A successful general fluid-to-fluid similarity theory for heat transfer at supercritical pressure. Int. J. Heat Mass Transf. 2020, 159, 120152. [CrossRef] [PubMed]

26. Kassem, S.; Pucciarelli, A.; Ambrosini, W. Insight into a fluid-to-fluid similarity theory for heat transfer at supercritical pressure: Results and perspectives. Int. J. Heat Mass Transf. 2021, 168, 120813. [CrossRef]

27. Watts, M.J. Heat Transfer to Supercritical Pressure Water-Mixed Convection with Upflow and Downflow in a Vertical Tube. Ph.D. Thesis, University of Manchester, Manchester, UK, 1980.

28. Kline, N. An Experimental Study on Heat Transfer Deterioration at Supercritical Pressures. Master's Thesis, Ottawa-Carleton Institute for Mechanical and Aerospace Engineering, University of Ottawa, Ottawa, ON, Canada, 2017.

29. He, J.; Yan, J.; Wang, W.; He, S. Direct Numerical Simulation Study for Fluid-To-Fluid Scaling for Fluids at Supercritical Pressure. In Proceedings of the 16th International Heat Transfer Conference, IHTC-16, Beijing, China, 10-15 August 2018. IHTC16-23265.

30. Kassem, S.; Pucciarelli, A.; Ambrosini, W. Outcomes of a Successful Fluid-To-Fluid Similarity Theory for Heat Transfer at Supercritical Pressures. In Proceedings of the 10th International Symposium on Supercritical Water-Cooled Reactors, ISSCWR-10, Prague, Czech Republic, 15-19 March 2021. ISSCWR-10-014.

31. Pucciarelli, A.; Kassem, S.; Ambrosini, W. Considerations on a Fluid-To-Fluid Scaling Theory for Heat Transfer to Supercritical Fluids: Recommendations for Non-Uniform Heat Flux Conditions. In Proceedings of the 10th International Symposium on Supercritical Water-Cooled Reactors, ISSCWR-10, Prague, Czech Republic, 15-19 March 2021. ISSCWR-10-012.

32. De Angelis, A.; Pucciarelli, A.; Kassem, S.; Ambrosini, W. Advances in the Development of a Fluid-To-Fluid Similarity Theory for Fluids at Supercritical Pressure: Results from Sensitivity Analyses. In Proceedings of the 2021 28th International Conference on Nuclear Engineering, ICONE28, online. 4-6 August 2021. ICONE28-64713.

33. Kassem, S.; Pucciarelli, A.; Ambrosini, W. Extending a Fluid-To-Fluid Similarity Rationale for Heat Transfer at Supercritical Pressure to R134a. In Proceedings of the 2021 28th International Conference on Nuclear Engineering, ICONE28, online. 4-6 August 2021. ICONE28-64822.

34. Cheng, H.; Zhao, J.; Rowinski, M.K. Study on two wall temperature peaks of supercritical fluid mixed convective heat transfer in circular tubes. Int. J. Heat Mass Transf. 2017, 113, 257-267. [CrossRef]

35. Ambrosini, W. On the Analogies in the Dynamic Behaviour of Heated Channels with Boiling and Supercritical Fluids. Nucl. Eng. Des. 2007, 237, 1164-1174. [CrossRef]

36. Ambrosini, W. Assessment of flow stability boundaries in a Heated Channel with Different Fluids at Supercritical Pressure. Ann. Nucl. Energy 2011, 38, 615-627. [CrossRef] 
37. Ambrosini, W. Discussion on the stability of heated channels with different fluids at supercritical pressures. Nucl. Eng. Des. 2009, 239, 2952-2963. [CrossRef]

38. De Angelis, A. Discussion and Further Assessment of a Fluid-To-Fluid Similarity Theory for Heat Transfer at Supercritical Pressures. Master's Thesis, Ingegneria Energetica, Università di Pisa, Pisa, Italy, 2019/2020. 